\title{
Photoelectric Dye Used for Okayama University-Type Retinal Prosthesis Reduces the Apoptosis of Photoreceptor Cells
}

\author{
Shihui Liu,, Toshihiko Matsuo,, Osamu Hosoya, and Tetsuya Uchida ${ }^{3}$
}

\begin{abstract}
Purpose: Our previous study demonstrated that photoelectric dye-coupled polyethylene film (Okayama University-type retinal prosthesis), which was implanted in subretinal space of the eyes of Royal College of Surgeons (RCS) rats, prevented retinal neurons from apoptotic death. In this study, we aimed to examine whether photoelectric dye itself would protect retinal neurons from apoptosis in RCS rats.

Methods: RCS rats received intravitreous injection of different concentrations of the dye in the left eye and housed under a 12-h light-dark cycle. Saline injection in the right eye served as control. In addition, RCS rats with dye injection were kept in 24-h daily dark condition. Sections were processed for terminal deoxynucleotidyl transferase-mediated fluorescein-conjugated-dUTP nick-end-labeling (TUNEL) assay and immunohistochemical staining of glial fibrillary acidic protein (GFAP) and protein kinase $\mathrm{C} \alpha(\mathrm{PKC} \alpha)$.

Results: The number of TUNEL-positive cells significantly decreased in the retina of dye-injected eyes compared with those in saline-injected eyes $(P=0.0001,2$-factor analysis of variance [ANOVA] $)$, under 12 -h light-dark cycle. Significant decrease of TUNEL-positive cells was noted in the retina of rats with dye injection compared with those with saline injection, kept under 24-h dark condition $(P=0.0001,2$-factor ANOVA). Immunoreactive area for GFAP decreased significantly in the retina of dye-injected eyes compared with that in controls ( $P=0.0001$, 2-factor ANOVA), whereas immunoreactive area for PKC $\alpha$ increased significantly in the retina of dye-injected eyes compared with that in controls $(P=0.01,2$-factor ANOVA).

Conclusions: Photoelectric dye inhibits apoptotic death of photoreceptor cells in RCS rats and downregulates GFAP expression in retinal Müller cells. Photoelectric dye may be a candidate agent for neuroprotection in retinitis pigmentosa and other retinal diseases.
\end{abstract}

Keywords: apoptosis, drug, retina, GFAP, $\mathrm{PKC} \alpha$, photoreceptors

\section{Introduction}

$\mathbf{R}$ ETINITIS PIGMENTOSA is a hereditary disease that involves the loss of retinal photoreceptor cells that perceive light. Patients with retinitis pigmentosa experience slowly progressive constriction of the visual field, caused by peripheral retinal dystrophy, and finally lose the vision by macular involvement. ${ }^{1}$ The speed of deterioration differs largely from patient to patient. ${ }^{2}$

In blind patients with retinitis pigmentosa who have lost all photoreceptor cells, treatment strategy is to replace dead photoreceptor cells with retinal prosthesis. ${ }^{3-5}$ Several types of retinal prosthesis have been developed recently to replace the lost photoreceptor cells. ${ }^{6-11}$ The prevailing types of retinal prosthesis output electric current in correspondence to cameracaptured and processed image, and stimulate the remaining retinal neurons that send axons to the brain. ${ }^{4,6}$ Other types of retinal prosthesis utilize photovoltaic systems ${ }^{7}$ or wireless subretinal neurostimulator. ${ }^{8}$ Our system used photoelectric dye molecules to replace dead photoreceptor cells. ${ }^{9-14}$

A photoelectric dye is an organic molecule that generates electric potential in response to light. ${ }^{10}$ Photoelectric dyecoupled polyethylene thin films (dye-coupled films), called

Departments of ${ }^{1}$ Ophthalmology and ${ }^{2}$ Medical Neurobiology, Okayama University Medical School and Graduate School of Medicine, Dentistry, and Pharmaceutical Sciences, Okayama City, Japan.

${ }^{3}$ Polymer Materials Science, Okayama University Faculty of Engineering and Graduate School of Natural Science and Technology, Okayama City, Japan.

(C) Shihui Liu, et al., 2017; Published by Mary Ann Liebert, Inc. This Open Access article is distributed under the terms of the Creative Commons Attribution Noncommercial License (http://creativecommons.org/licenses/by-nc/4.0/) which permits any noncommercial use, distribution, and reproduction in any medium, provided the original author(s) and the source are credited. 
Okayama University-type retinal prosthesis (OUReP $\left.{ }^{\mathrm{TM}}\right)$, induced neuronal response at light stimulation. ${ }^{12,13}$ Our previous studies showed that OUReP could reduce neuronal apoptosis in the eyes of Royal College of Surgeons (RCS) rats. ${ }^{15,16}$ In this study, we tested whether intravitreous injection of the photoelectric dye would rescue photoreceptor cells from the apoptosis in RCS rats. ${ }^{17-19}$

\section{Methods}

\section{Animals and experimental design}

Photoelectric dye molecules, 2-[2-[4-(dibutylamino) phenyl] ethenyl]-3-carboxymethylbenzothiazolium bromide (503.5 of molecular weight, NK-5962, Hayashibara, Inc., Okayama, Japan, Fig. 1A), were dissolved in distilled deionized water (Fig. 1B) at a concentration of $8.2 \mu \mathrm{g} / \mathrm{mL}$ $(16 \mu \mathrm{M})$. This original stock solution $(8.2 \mu \mathrm{g} / \mathrm{mL})$ was diluted with saline $(0.9 \%$ sodium chloride $)$ to make a series of 10 -fold dilutions from $8.2 \times 10^{-4}$ to $8.2 \mu \mathrm{g} / \mathrm{mL}$.
Twenty male pink-eyed RCS rats (RCS/Jcl-rdy/rdy, p-, CLEA Japan, Inc., Tokyo) at the age of 4 weeks were assigned to 5 groups $(n=4)$ that received the dye at 5 different concentrations. Intravitreous injection was done twice at the age of 4 and 5 weeks (Fig. 1D). This study was approved by the Animal Care and Use Committee at Okayama University, based on the Animal Welfare and Management Act in Japan. All experimental procedures were in accordance with the ARVO statement for the use of animals in ophthalmic and vision research.

RCS rats were anesthetized by intraperitoneal injection of ketamine $(87 \mathrm{mg} / \mathrm{kg}$ body weight $)$ and xylasine $(13 \mathrm{mg} / \mathrm{kg})$. Mydriasis was induced by $0.5 \%$ tropicamide and $0.5 \%$ phenylephrine instillation, and corneal anesthesia was further obtained with $0.4 \%$ oxybuprokaine instillation. Under a dissecting microscope, $3 \mu \mathrm{L}$ of dye solution and saline was injected into the vitreous of the left eye and the right eye, respectively, with a 30-gauge needle attached to a Hamilton syringe $(50 \mu \mathrm{L} 1705$ LT SYR; Hamilton Company, Reno,
FIG. 1. Experimental design. (A) Molecular structure of photoelectric dye 2-[2-[4-(dibutylamino) phenyl] ethenyl]3-carboxymethylbenzothiazolium. (B) Stock solution of the dye dissolved in water at a concentration of $8.2 \mu \mathrm{g} / \mathrm{mL}$ $(16 \mu \mathrm{M})$. (C) Four retinal sites defined for immunohistochemical analysis. "a" and " $b$ " begin at the straight distance of 373 and $160 \mu \mathrm{m}$, respectively, superior from the optic nerve head. "c" and " $d$ " begin at the straight distance of 160 and $373 \mu \mathrm{m}$, respectively, inferior from the optic nerve head. (D) Time table for injections.
A<smiles>CCCCCCCCCCCCN(CCCCCC)c1ccc(C=Cc2sc3ccccc3[n+]2CC(=O)O)cc1</smiles>

C

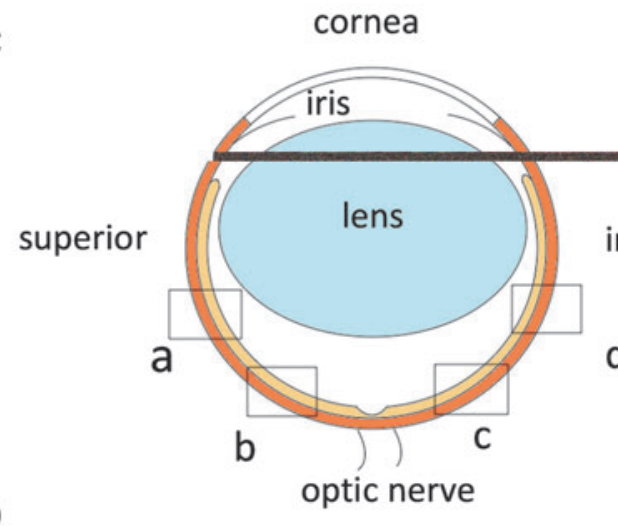

inferior
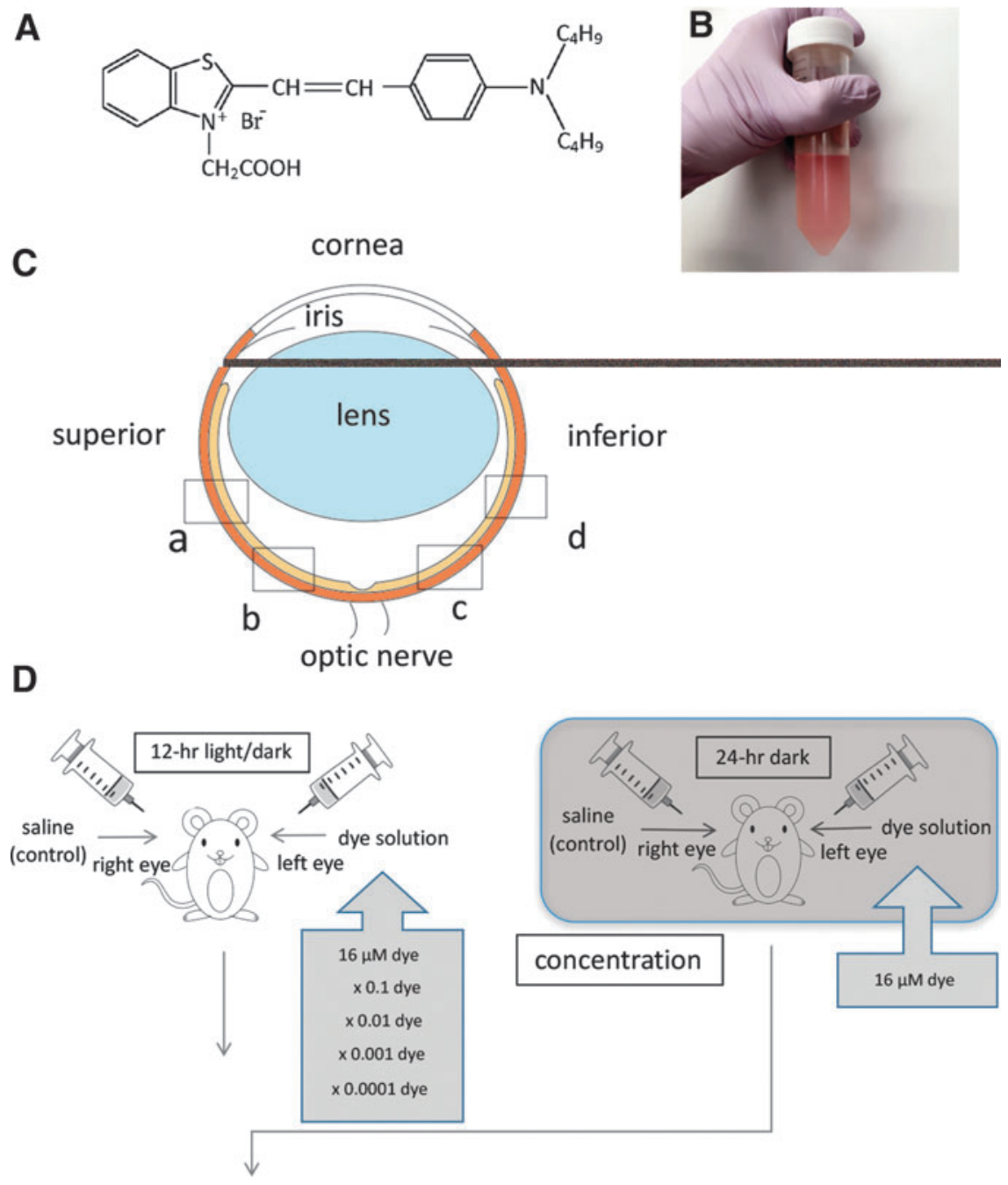

age:

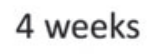

5 weeks

6 weeks
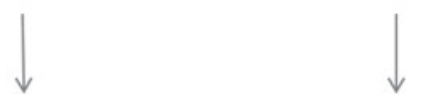

intravitreous injection

intravitreous injection 


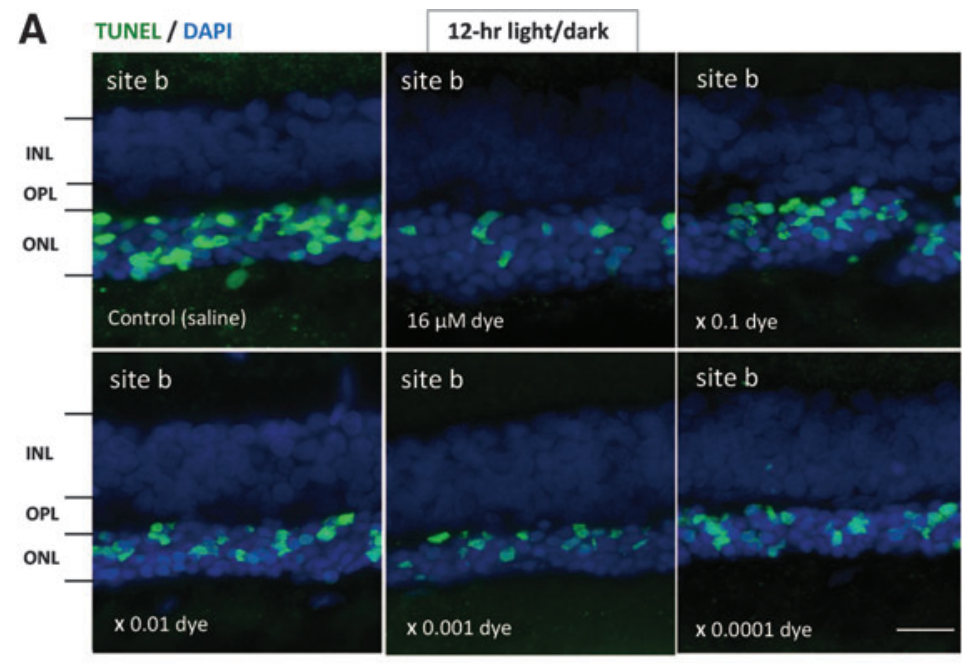

B TUNEL/DAPI

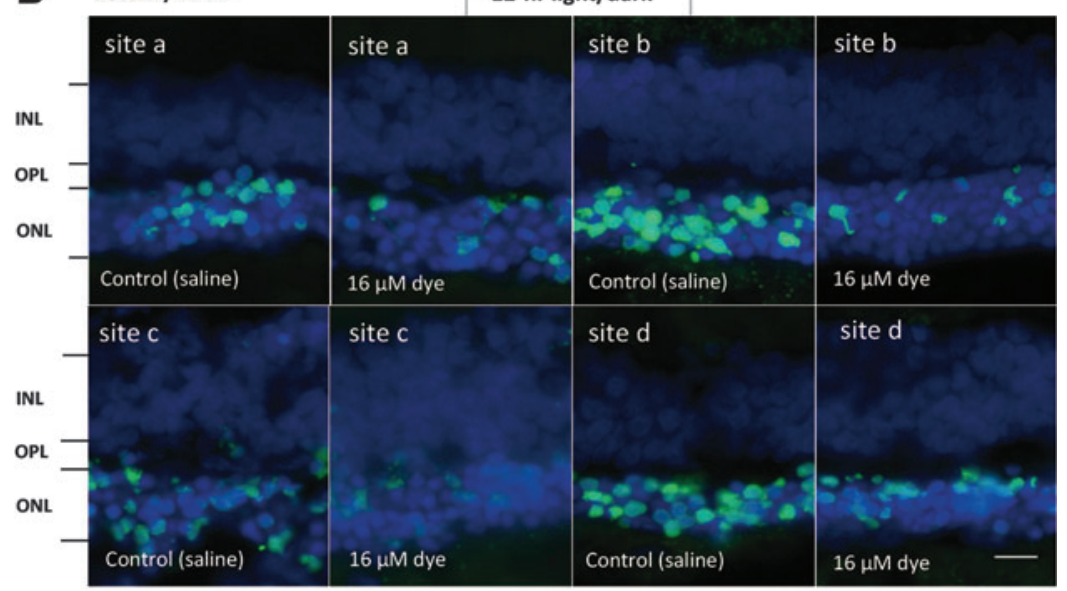

C TUNEL/DAPI

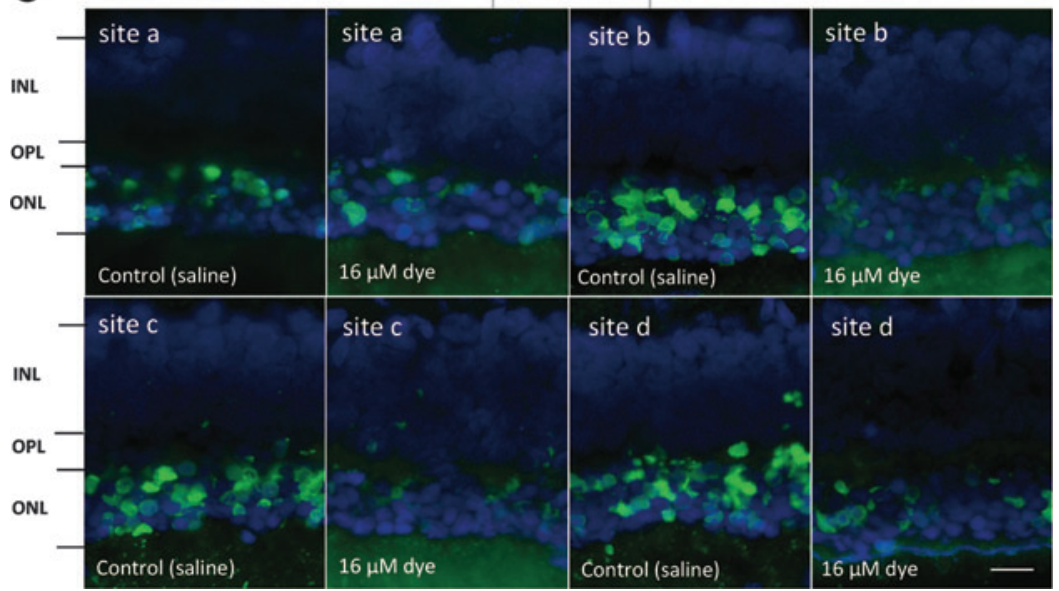

FIG. 2. The detection of apoptosis in retinal sections of each group of rats. (A) TUNEL staining (green) of retinal sections (site b) of the eyes with intravitreous injection of $3 \mu \mathrm{L}$ saline in the right eye or series of dilutions of the dye stock solution in the left eye under 12-h light-dark cycle. Eyes were enucleated 2 weeks after the first injection at the age of 4 weeks. The nuclei were counterstained with DAPI (blue). The number of TUNEL-positive cells in the outer nuclear layer (ONL) was less in the dye-injected eyes than in the saline-injected eyes. (B) TUNEL staining of retinal sections at 4 different retinal sites $(a, b, c$, and d) in the left eye with dye injection $(16 \mu \mathrm{M})$ compared with that in the right eye with saline injection under 12-h light-dark cycle. (C) TUNEL staining of retinal sections at 4 different retinal sites (a, b, c, and d) in the left eye with dye injection $(16 \mu \mathrm{M})$ compared with that in the right eye with saline injection under 24-h constant dark condition. INL, inner nuclear layer; OPL, outer plexiform layer. Scale bar: $10 \mu \mathrm{m}$. DAPI, 4',6diamidino-2-phenylindole.
NV). The needle was inserted almost at a perpendicular angle, $1 \mathrm{~mm}$ from the corneoscleral limbus to avoid the damage to the lens. Next, antibiotic ( $0.5 \%$ moxifloxacin) eye drops were instilled to both eyes. Each rat was housed in a standard rat cage in the 12-h each light and dark cycle at the Animal Center of Okayama University. In a separate experiment, 3 rats were placed and maintained in constant darkness after intravitreous injection of the dye at a concentration of $8.2 \mu \mathrm{g} / \mathrm{mL}(16 \mu \mathrm{M})$.

\section{Immunohistochemistry}

At the age of 6 weeks, 2 weeks after the first injection, rats were sacrificed by an overdose of ether. The eyes were enucleated and fixed in $4 \%$ paraformaldehyde for $2-3 \mathrm{~h}$, cut into halves circumferentially in the midperiphery of the eye balls, and cryoprotected by immersion in $10 \%$ sucrose in $100 \mathrm{mM}$ phosphate buffer ( $\mathrm{pH}$ 7.4) for $3 \mathrm{~h}$. Serial frozen sections with $14 \mu \mathrm{m}$ thickness were cut parallel to the vertical meridian of 
FIG. 3. Quantitative analysis of apoptotic cells in the ONL of each group of rats. TUNEL-positive cell counts per $1,000 \mu \mathrm{m}^{2}$ in the ONL of 4 different retinal sites $(\mathrm{a}, \mathrm{b}, \mathrm{c}$, and d) of the left eye with dye injection at 5 concentrations compared with those of the right eye with saline injection under 12-h light-dark cycle. TUNEL-positive cell counts were significantly different among different dye concentrations $(P=0.0001)$, but not significantly different among 4 different retinal sites $(P=$ 0.144 , 2-factor analysis of variance [ANOVA]). TUNELpositive cell counts in dyeinjected eyes were significantly less than in saline-injected eyes at the concentration of $16 \mu \mathrm{M}$ $(* * * P=0.0001), \quad 16 \mu \mathrm{M} \times 0.1$ $(* * * P=0.0001)$, and $16 \mu \mathrm{M} \times$ $0.01(* * P=0.002)$. The bottom right panel shows TUNELpositive cell counts in the eyes injected with dye $(16 \mu \mathrm{M})$ versus saline under 12-h lightdark cycle versus under 24-h constant dark condition. There was significant difference between dye-injected eyes and saline-injected eyes under 24-h dark condition $(* * * P=0.0001)$. $\mathrm{T}$ bars indicate standard deviation.
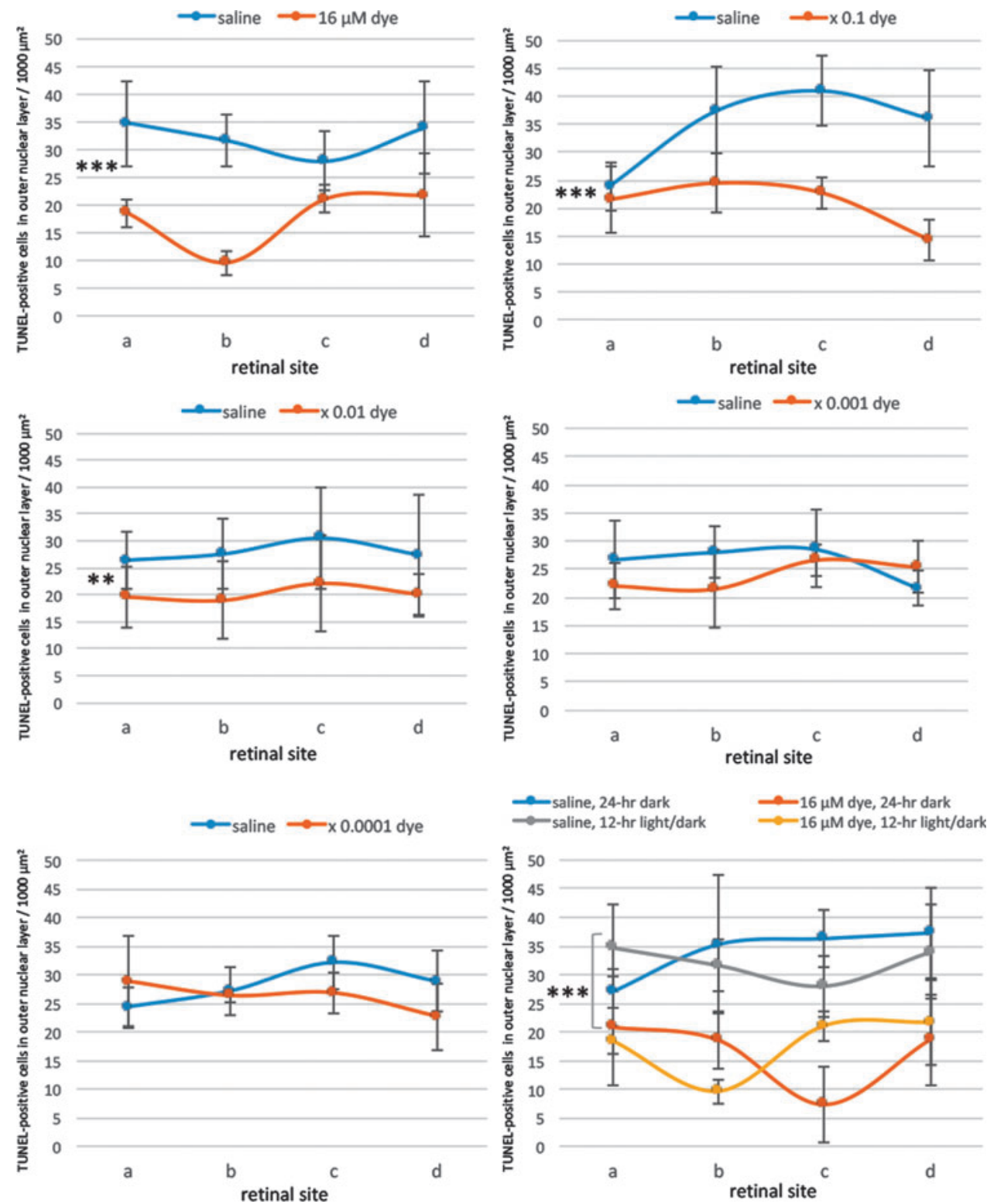

the eye at the optic nerve head, and immunohistochemical staining was done as described previously. ${ }^{14-16}$ The primary antibodies were antiglial fibrillary acidic protein (GFAP) in 1:200 dilution (mouse monoclonal antibody, Chemicon, Temecula, $\mathrm{CA})$ and antiprotein kinase $\mathrm{C} \alpha(\mathrm{PKC} \alpha)$ in 1:250 dilution (mouse monoclonal antibody; Sigma-Aldrich, St. Louis, MO). Alexa Fluor 594 goat antimouse IgG was used as a fluorescence-conjugated secondary antibody. Nuclei were counterstained with 4',6-diamidino-2-phenylindole (DAPI). The tissue was covered with antifade reagents (ProLong Antifade Kit; Invitrogen, Waltham, MA) and air dried.

All images were captured at the constant exposure time with a frame $(438 \mu \mathrm{m} \times 330 \mu \mathrm{m})$ by an Olympus FSX100 microscope, using $20 \times$ NA0.75 objective lens (Olympus, Tokyo, Japan), and saved as 24-bit RGB TIFF files. Alexa Fluor 594 and DAPI signals were sequentially detected with Olympus filter sets U-MWIG3 and U-MNUA2. GFAP or $\mathrm{PKC} \alpha$ immunoreactivity was assessed in 4 retinal sites: "a" and " $\mathrm{d}$," beginning at $373 \mu \mathrm{m}$ superiorly and inferiorly from the optic nerve head, respectively, and sites " $b$ " and "c," beginning at $160 \mu \mathrm{m}$ superiorly and inferiorly from the optic nerve head, respectively (Fig. 1C). Five photographs in each of 5 retinal vertical sections per eye were taken, and an entire frame of each photograph with $20 \times$ objective lens was used for the following measurements.

RGB images were imported into the Image $\mathrm{J}$ software (National Institutes of Health, Bethesda, MD), and converted to 8-bit grayscale images. For background subtraction, the mean background staining was defined by measuring an area positioned away from specific immunoreactive signals. After the background subtraction, the total area of immunoreactivity was determined by using a standardized histogram-based technique. To define a pixel as immunoreactive, constant threshold values were set to 50 for GFAP and 55 for $\mathrm{PKC} \alpha$ immunoreactivity, and the number of pixels that matched or exceed each set threshold value was calculated for each image. Finally, the percentage of immunoreactive area was calculated from the total immunoreactive area in a given size frame (height $193 \mu \mathrm{m}$, width $173 \mu \mathrm{m}$ ) at 4 predefined sites per retinal section (sites 

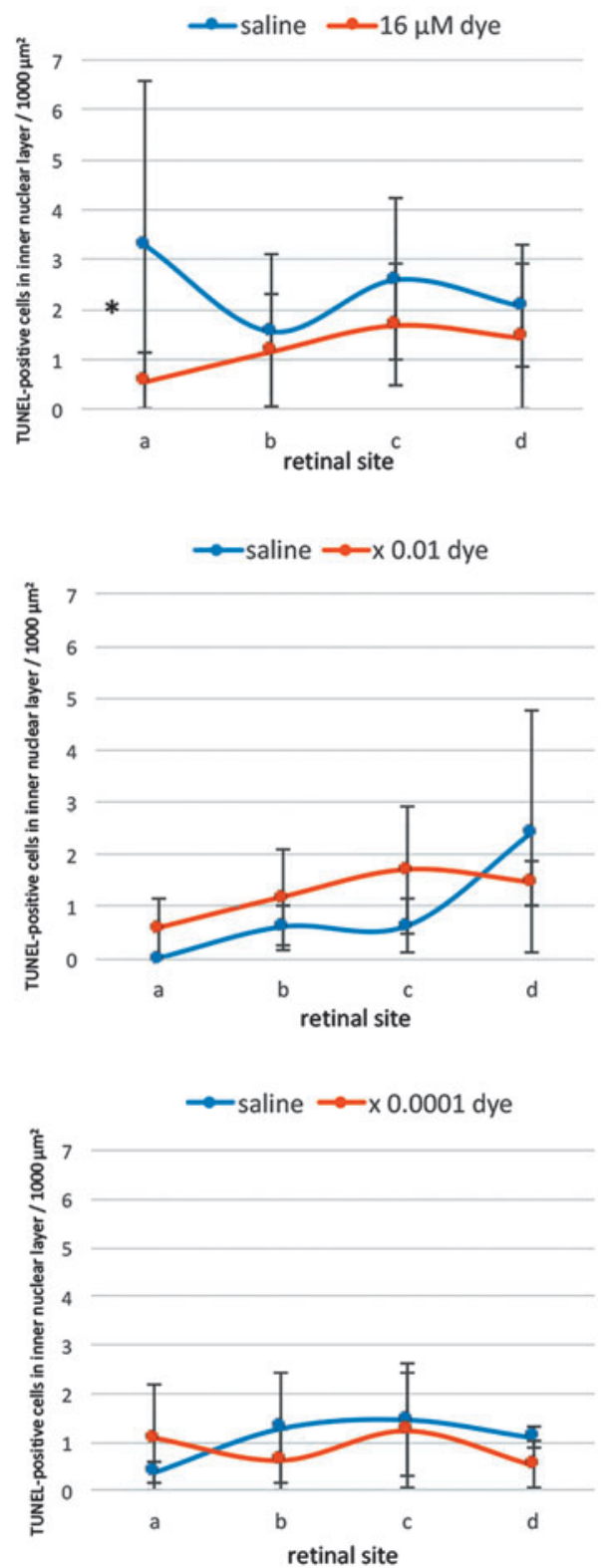

FIG. 4. Quantitative analysis of apoptotic cells in the inner nuclear layer of each group of rats. TUNELpositive cell counts per $1,000 \mu \mathrm{m}^{2}$ in the inner nuclear layer of 4 different retinal sites (a, b, c, and d) of the left eye with dye injection at 5 concentrations compared with those of the right eye with saline injection under 12 -h light-dark cycle. TUNELpositive cell counts showed no significant differences among different dye concentrations $\quad(P=0.084) \quad$ and among 4 different retinal sites $(P=0.927, \quad 2$-factor ANOVA). However, TUNELpositive cell counts in dyeinjected eyes were significantly less than in salineinjected eyes at the concentration of $16 \mu \mathrm{M}(* P=0.043$, post hoc test). The bottom right panel shows TUNELpositive cell counts in the eyes injected with dye $(16 \mu \mathrm{M})$ versus saline under 12-h light-dark cycle versus under 24-h constant dark condition. There was no significant difference between dye-injected eyes and salineinjected eyes under 24-h dark condition. $\mathrm{T}$ bars indicate standard deviation. $\mathrm{a}, \mathrm{b}, \mathrm{c}$, and d), and the data were compared by 2-factor analysis of variance (ANOVA).

\section{Apoptosis detection and retinal thickness measurement}

Apoptotic cells were detected by terminal deoxynucleotidyl transferase-mediated fluorescein-conjugateddUTP nick-end-labeling (TUNEL) assay, according to the manufacturer's instructions (In Situ Cell Death Detection Kit, Roche Diagnostics, Mannheim, Germany). The images at the constant exposure time were captured by an Olympus FSX100 microscope, equipped with the fluorescent filter cube U-MWIBA3. The areas for TUNEL assay were the same as those for immunohistochemical staining (sites $a, b$, $\mathrm{c}$, and d). Five photographs in each of 5 retinal vertical sections per eye were taken, and an entire frame $(220 \times 165 \mu \mathrm{m})$ of each photograph with $40 \times$ NA0.95 objective lens was used for measurements. The number of
TUNEL-positive cells in the inner nuclear layer and the outer nuclear layer was counted in each frame of photographs, and the thickness of the inner and outer nuclear layer was measured. The number of TUNEL-positive cells per $1,000 \mu \mathrm{m}^{2}$ of the inner nuclear layer or the outer nuclear layer was calculated for comparison by 2-factor ANOVA.

\section{Statistical analysis}

All data were presented as mean \pm standard deviation and analyzed by 2 -factor ANOVA followed by post hoc test if appropriate. $P<0.05$ was accepted as statistically significant.

\section{Results}

\section{TUNEL staining and retinal thickness}

TUNEL-positive signals were compared among the left eyes with dye injection at different concentrations and 
among the right eyes with control saline injection. Under 12-h light-dark cycle condition, in saline-injected eyes, numerous TUNEL-positive signals were detectable in the outer nuclear layer where the nuclei of photoreceptor cells were located. In contrast, TUNEL-positive cells appeared to decrease in all 4 different sites (a, b, c, and d) of retinal sections from dye-injected eyes (Fig. 2). TUNEL-positive cells in the outer nuclear layer were significantly different among different concentrations of the dye $(P=0.0001)$, but not significantly different among 4 different retinal sites $(P=0.144$, 2-factor ANOVA, Fig. 3). TUNEL-positive cells in the outer nuclear layer of dye-injected eyes were significantly less at 3 higher concentrations of the dye, ranging from 16 to $16 \times 10^{-2} \mu \mathrm{M}$, compared with those of salineinjected eyes $(P=0.0001, P=0.0001$, and $P=0.002$, post hoc test, the least significant difference, Fig. 3).

In contrast, the number of TUNEL-positive cells in the inner nuclear layer was significantly different neither among different concentrations of the dye $(P=0.084)$ nor among 4 different retinal sites $(P=0.927,2$-factor ANOVA, Fig. 4). However, post hoc test showed significant decrease of the number of TUNEL-positive cells in the inner nuclear layer only at the highest concentration of the dye $(16 \mu \mathrm{M})$ compared those with saline ( $P=0.043$, Fig. 4$)$.

Morphometric analysis showed that the thickness of the outer nuclear layer was significantly different among different concentrations of the dye $(P=0.0001)$ and among 4 different retinal sites ( $P=0.014$, 2-factor ANOVA, Fig. 5). The outer nuclear layer was significantly thicker in the dyeinjected eyes at higher concentrations, ranging from 16 to $16 \times 10^{-2} \mu \mathrm{M}$, than that in saline-injected eyes $(P=0.001$, $P=0.001$, and $P=0.026$, post hoc test, Fig. 5), under 12-h light-dark cycle condition. In addition, significant difference was noted in the thickness of the inner nuclear layer among different concentrations of the dye $(P=0.014)$ and among 4 different retinal sites $(P=0.0001,2$-factor ANOVA, Fig. 6), under 12-h light-dark cycle condition. However, post hoc tests did not reach significance.

In another series of experiments, RCS rats injected with saline and dye at a highest concentration $(16 \mu \mathrm{M})$ were kept under constant dark condition. The number of TUNELpositive cells in the outer nuclear layer was significantly
FIG. 5. Quantitative analysis of the thickness of the ONL of each group of rats. The thickness of the ONL of 4 different retinal sites (a, b, c, and d) of the left eye with dye injection at 5 concentrations compared with that of the right eye with saline injection under 12-h light-dark cycle. The thickness showed significant differences among different concentrations of the dye $(P=0.0001)$ and among 4 different retinal sites $(P=0.014$, 2-factor ANOVA). The ONL was significantly thicker with dye injection at 3 concentrations, $16 \mu \mathrm{M} \quad(* * P=0.001)$, $16 \mu \mathrm{M} \times 0.1(* * P=0.001)$, and $16 \mu \mathrm{M} \times 0.01 \quad(* P=0.026)$, than with saline injection. The bottom right panel shows the ONL thickness of the eyes injected with dye $(16 \mu \mathrm{M})$ versus saline under 12-h light-dark cycle versus under 24-h constant dark condition. There was no significant difference between dye-injected eyes and saline-injected eyes under 24-h dark condition. T bars indicate standard deviation.
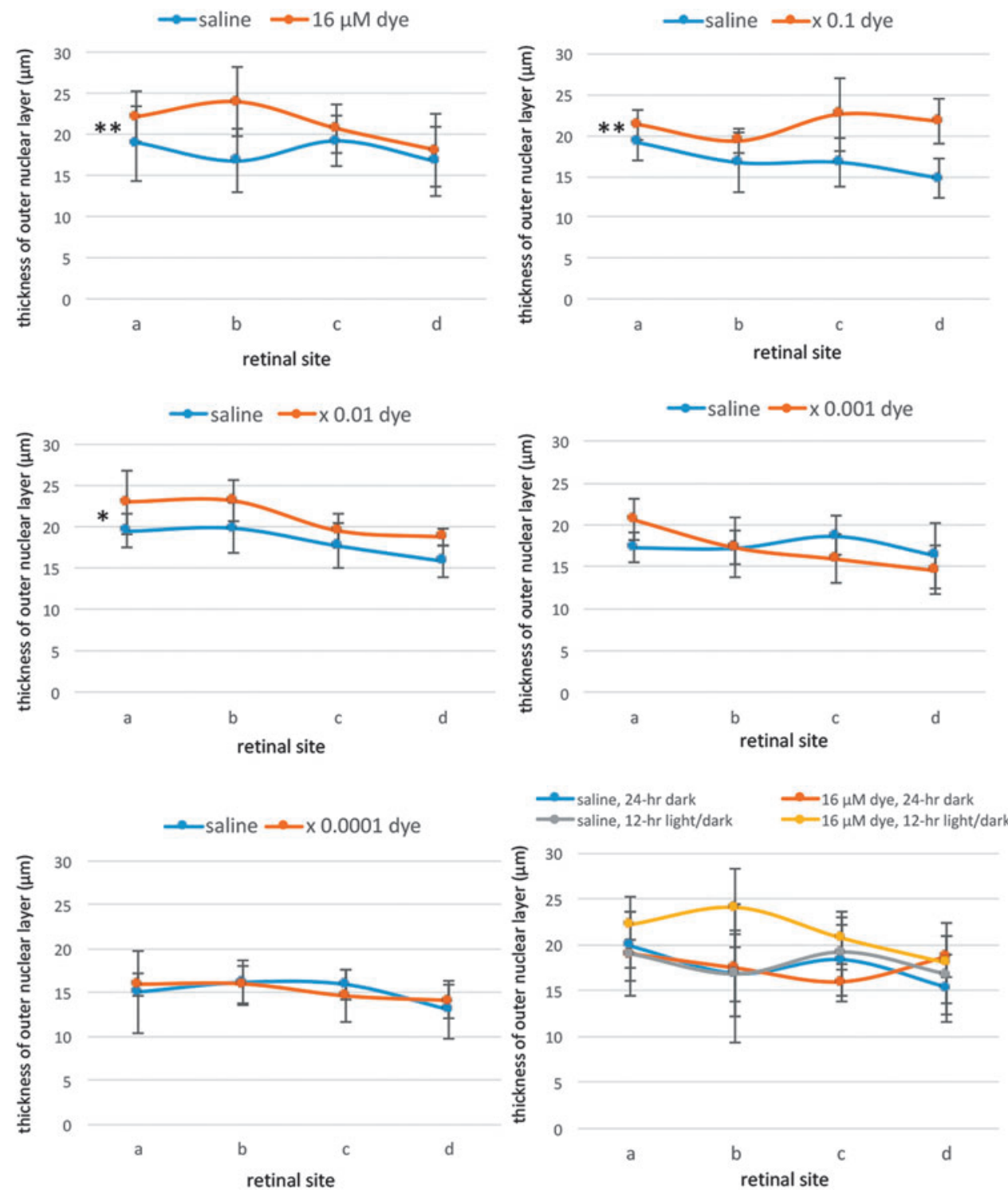


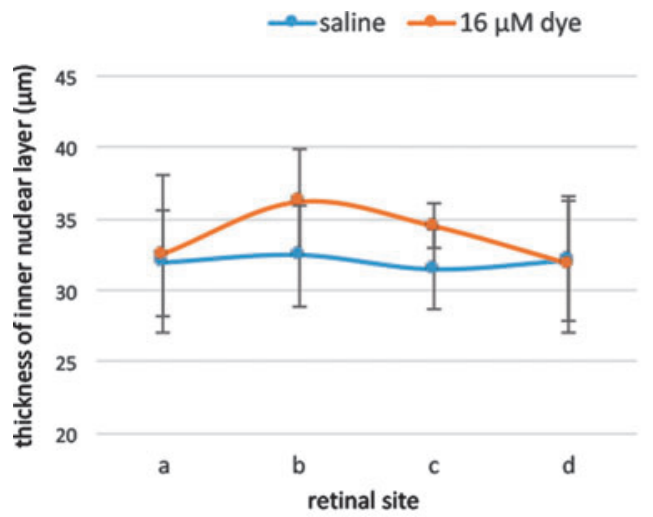

$\rightarrow$ saline $\rightarrow x 0.01$ dye
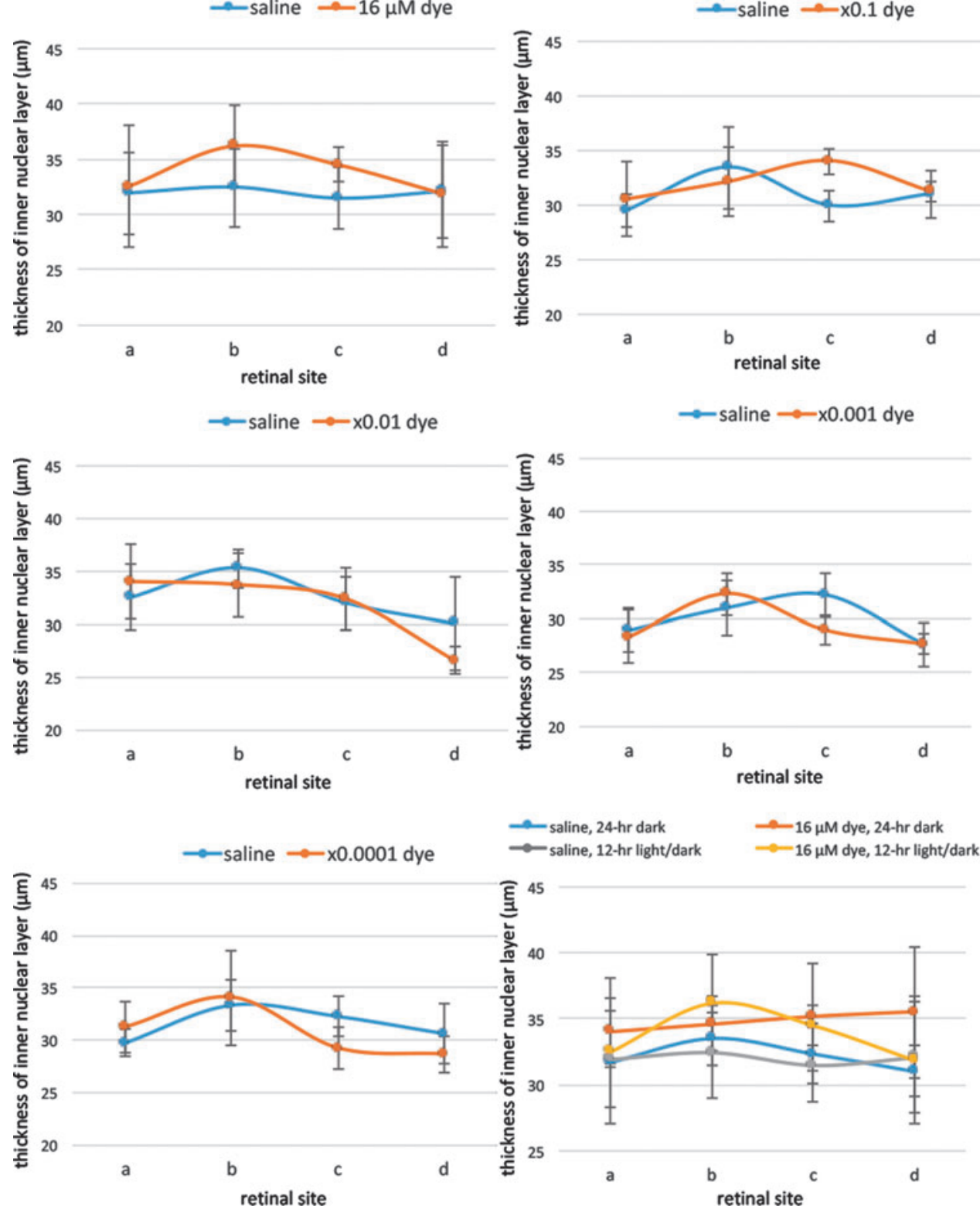

FIG. 6. Quantitative analysis of the thickness of the inner nuclear layer of each group of rats. The thickness of the inner nuclear layer of 4 different retinal sites $(\mathrm{a}, \mathrm{b}, \mathrm{c}$, and d) of the left eye with dye injection at 5 concentrations compared with that of the right eye with saline injection under 12-h light-dark cycle. The thickness showed significant differences among different concentrations of the dye $(P=0.014)$ and among 4 different retinal sites $(P=0.0001, \quad 2$-factor ANOVA). However, post hoc test showed no significance at each concentration of the dye compared with that of saline. The bottom right panel shows the inner nuclear layer thickness of the eyes injected with dye $(16 \mu \mathrm{M})$ versus saline under 12-h light-dark cycle versus under 24-h constant dark condition. There was no significant difference between dye-injected eyes and salineinjected eyes under 24-h dark condition. $\mathrm{T}$ bars indicate standard deviation. different between dye injection and saline injection $(P=0.0001)$, but not significantly different among 4 different retinal sites $(P=0.982,2$-factor ANOVA). As post hoc tests, the number of TUNEL-positive cells in the outer nuclear layer of the dye-injected eyes was significantly less than in the saline-injected eyes $(P=0.0001)$, but no significant difference was noted in the number of TUNELpositive cells between the constant dark condition and the 12-h light-dark cycle ( $P=0.411$, Figs. 2 and 3$)$. The number of TUNEL-positive cells in the inner nuclear layer showed no significant difference between the dye-injected eyes and saline-injected eyes (Fig. 4).

Morphometric analysis showed that the outer nuclear layer tended to be thicker in the dye-injected eyes than in the saline-injected eyes under constant dark condition, although the difference was not significant $(P=0.054$, Fig. 5). No significant difference was noted in the thickness of the inner nuclear layer between the dye-injected and saline-injected eyes, under constant dark cycle condition $(P=0.145$, Fig. 6).

\section{GFAP immunohistochemistry}

GFAP-immunoreactive signals were compared among the left eyes with dye injection at different concentrations and among the right eyes with control saline injection. In the saline-injected eyes, strong GFAP-immunoreactivity was present at Müller cells in all sites of the retina and was mainly localized on their end feet that spread to the ganglion cell layer. In addition, radial Müller cell processes, running in the inner plexiform layer, showed intense GFAP immunofluorescence (Fig. 7). In the dye-injected eyes, immunohistochemical localization of GFAP in the retina was basically similar to that observed in the saline-injected eyes (Fig. 7). At a closer look, many Müller cell radial processes, exhibiting weak and discontinuous staining, were noted in the inner plexiform layer (Fig. 7).

Semiquantitative analysis showed that GFAP-immunoreactive areas were significantly different among different concentrations of the dye $(P=0.0001)$ and also among 4 different retinal sites $(P=0.0001,2$-factor ANOVA, Fig. 8). GFAP-immunoreactive 
areas significantly decreased at 2 concentrations of the dye (16 and $\left.16 \times 10^{-2} \mu \mathrm{M}\right)$ in the dye-injected eyes compared with those in the saline-injected eyes $(P=0.023$ and $P=0.007$, post hoc test, Fig. 8).

\section{$P K C \alpha$ immunohistochemistry}

In dye-injected eyes, PKC $\alpha$ immunoreactivity was evident in dendritic terminals of rod bipolar cells in the outer
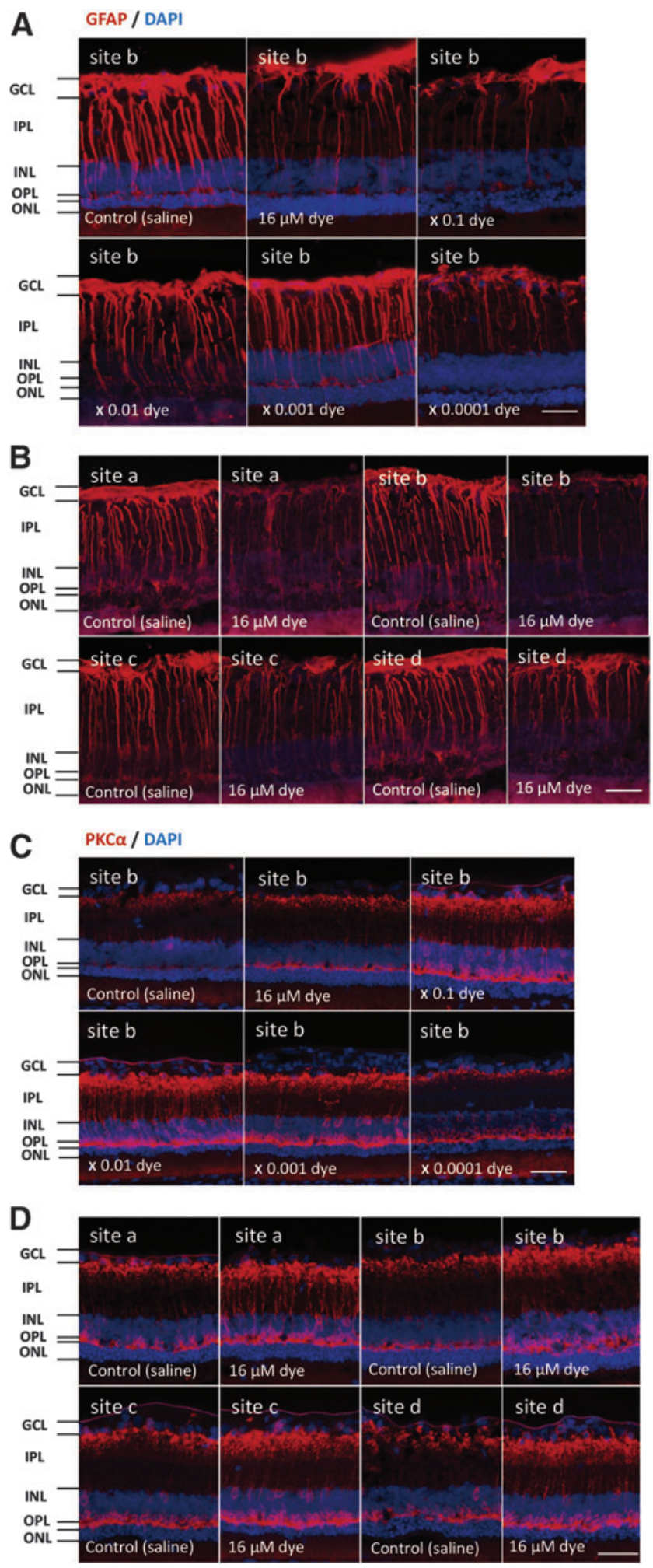

plexiform layer, somata in the inner nuclear layer, and axons, running perpendicularly throughout the inner plexiform layer, as well as the axon terminals located at the border between the inner plexiform layer and ganglion cell layer. The patterns of $\mathrm{PKC} \alpha$ staining in the retina of the dyeinjected eyes appeared to be different from those in the saline-injected eyes (Fig. 7). PKC $\alpha$-immunoreactive areas were significantly different among different concentrations of the dye $(P=0.01)$, but not significantly different among 4 different retinal sites ( $P=0.413,2$-factor ANOVA, Fig. 9). Post hoc tests showed significant increase of PKC $\alpha$ immunoreactive areas at 3 higher dye concentrations, ranging from 16 to $16 \times 10^{-2} \mu \mathrm{M}$, compared with those at saline injection $(P=0.002, P=0.003$, and $P=0.014$, respectively).

\section{Discussion}

This study aimed to investigate whether the photoelectric dye has protective effect on retinal neurons. We used RCS rats as an animal model, which show progressive photoreceptor degeneration as the consequence of a primary retinal pigment epithelium gene mutation. ${ }^{20}$ Our results demonstrated that repeated intravitreous injection of the photoelectric dye provided the protection of the outer nuclear layer neurons at the earlier stage of dystrophy in RCS rats both under the 12-h light-dark cycle and under the 24-h continuous dark condition.

Many potential agents have been tested in animal models for their ability to protect photoreceptor cells from death. ${ }^{21-23}$ Ciliary neurotrophic factor has entered into a phase I clinical trial in human patients with retinitis pigmentosa. ${ }^{24}$ Our previous studies showed that the photoelectric dye-coupled films (OUReP) could reduce neuronal apoptosis. ${ }^{15,16}$ In addition, the photoelectric dye could prevent retinal cells from death in mixed culture of glial cells and retinal neurons in vitro. ${ }^{25} \mathrm{We}$, therefore, speculated that the photoelectric dye might become a new neuroprotective agent.

Because the lens is relatively large in the small space of the eyeball of the rats, repeated intraocular injections would

FIG. 7. Immunohistochemical staining of GFAP and PKC $\alpha$ in each group of rats. (A) GFAP staining (red) of the retina (site " $b$ ") of RCS rats 2 weeks after intravitreous injection. The right eyes had saline injection and the left eyes had photoelectric dye injection at each concentration of 10 -fold dilution series from $16 \mu \mathrm{M}$. (B) GFAP staining (red) of the retina at 4 different retinal sites (a, b, c, and d) in the left eye with dye injection $(16 \mu \mathrm{M})$ compared with that in the right eye with saline injection under 12-h light-dark cycle. GFAP was downregulated with photoelectric dye injection. (C) PKC $\alpha$ staining (red) of the retina (site " $b$ ") of RCS rats 2 weeks after intravitreous injection. The right eyes had saline injection and the left eyes had photoelectric dye injection at each concentration of 10-fold dilution series from $16 \mu \mathrm{M}$. (D) $\mathrm{PKC} \alpha$ staining (red) of the retina at 4 different retinal sites (a, b, c, and d) in the left eye with dye injection $(16 \mu \mathrm{M})$ compared with that in the right eye with saline injection under 12-h light-dark cycle. Dye injection led to enhanced staining in rod bipolar cells including their dendrites, soma, and axon terminals. The nuclei were counterstained with DAPI (blue). GCL, ganglion cell layer; IPL, inner plexiform layer; INL, inner nuclear layer; OPL, outer plexiform layer. Scale bar: $30 \mu \mathrm{m}$. GFAP, glial fibrillary acidic protein; $\mathrm{PKC} \alpha$, protein kinase $\mathrm{C} \alpha$; $\mathrm{RCS}$, Royal College of Surgeons. 

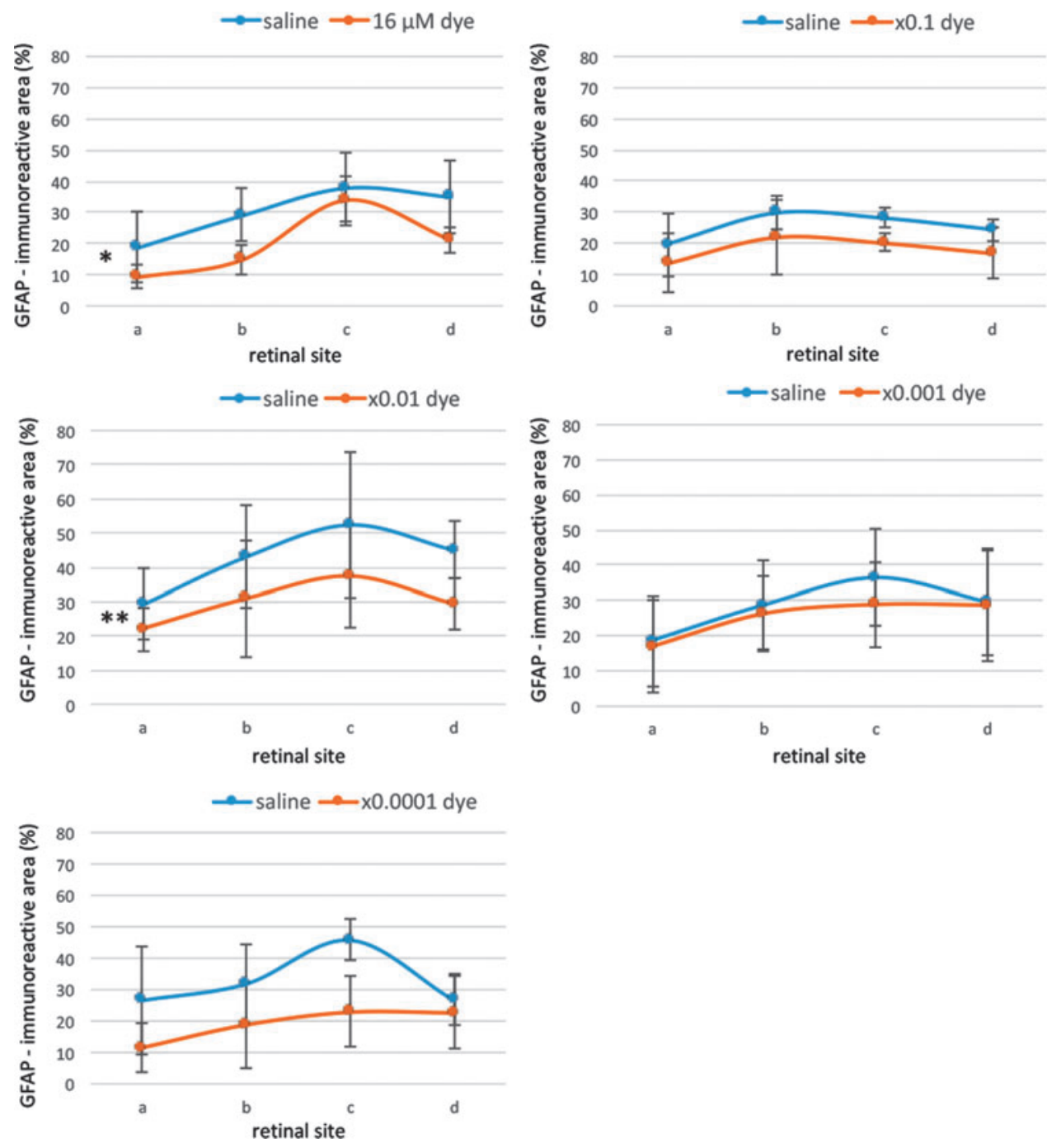

FIG. 8. Semiquantitative analysis of GFAP staining. GFAP immunoreactive areas were calculated for 4 different retinal sites $(a, b$, c, d) of the left eyes with dye injection at different concentrations and the right eyes with saline injection. GFAP staining showed significant differences among different concentrations of the dye $(P=0.0001)$ and among 4 different retinal sites $(P=0.0001,2$-factor ANOVA). GFAP staining was significantly less with dye injection at $16 \mu \mathrm{M}$ $(* P=0.023)$ and $16 \mu \mathrm{M} \times$ $0.01(* * P=0.007)$ than with saline injection. $\mathrm{T}$ bars indicate standard deviation. cause cataract formation and vitreous hemorrhage. We took care to reduce the incidence of cataract formation and vitreous hemorrhage after intravitreous injection. ${ }^{26}$ In this study, the photoelectric dye was administered intravitreally twice at 4 and 5 weeks of the age in RCS rats, and the rats were observed in the following 7 days. In the strain of RCS rats, used in this study, photoreceptor cells begin to degenerate on the 22nd day after birth, and the degeneration proceeds rapidly toward the 32 nd day. ${ }^{17}$ In a previous study, TUNEL-labeled photoreceptor cells suddenly appeared during 25-30 days, and the majority of the photoreceptor nuclei were TUNEL labeled on 35 days of age. ${ }^{18}$ Few photoreceptor nuclei remain in the outer nuclear layer by the 60th day. ${ }^{8,19} \mathrm{We}$, therefore, chose the age of fourth week for the timing of injection to determine whether the photoelectric dye was effective to prevent photoreceptor cells from apoptotic death.

The treatment with the dye under 24-h continuous dark condition also significantly reduced apoptotic cells. Furthermore, we found no difference in the number of retinal apoptotic cells between the 24-h continuous dark condition and the 12-h light-dark cycle. However, a previous study reported that dark condition could delay the deterioration of the dystrophic rat retina and that the animals in a dark environment showed less deterioration of the electroretinographic response. ${ }^{17}$ Further studies would be required to explain the contradictory results between the previous study $^{17}$ and this study, concerning the reduction of retinal apoptosis under constant dark and light-dark cycle condition.

GFAP is a constituent of glial filaments in astrocytes. In the central nervous system, astrocytes respond in an active manner when they suffer from diseases, trauma, chemical insults, and genetic disorders. ${ }^{27}$ Enhanced expression of GFAP is considered a marker of gliosis and hypertrophy of glial cells. ${ }^{28-30}$ Therefore, we examined GFAP immunoreactivity in retinal Müller cells after intravitreous dye injection. We found that intravitreous injection of the dye led to direct or indirect downregulation of GFAP expression, and might inhibit glial injury reaction. The variability in the semiquantitative image analysis resulted in significant differences in GFAP staining for some concentrations of the dye, but not for others. Nevertheless, the results suggest that photoelectric dye may downregulate GFAP expression and inhibit glial cell activation.

Rod bipolar cells are second-order retinal neurons that make synapses with rod photoreceptors, and their axon terminals are located in the inner plexiform layer, close to 
FIG. 9. Semiquantitative analysis of $\mathrm{PKC} \alpha$ staining. $\mathrm{PKC} \alpha$ immunoreactive areas were calculated for 4 different retinal sites (a, b, c, and d) of the left eyes with dye injection at different concentrations and the right eyes with saline injection. PKC $\alpha$ staining showed significant differences among different concentrations of the dye $(P=0.01)$, but not among 4 different retinal sites $(P=$ 0.413 , 2-factor ANOVA). $\mathrm{PKC} \alpha$ staining was significantly higher with dye injection at $16 \mu \mathrm{M}(* * P=0.002)$, $16 \mu \mathrm{M} \times 0.1(* * P=0.003)$, and $16 \mu \mathrm{M} \times 0.01(* P=0.014)$ than with saline injection. $\mathrm{T}$ bars indicate standard deviation.
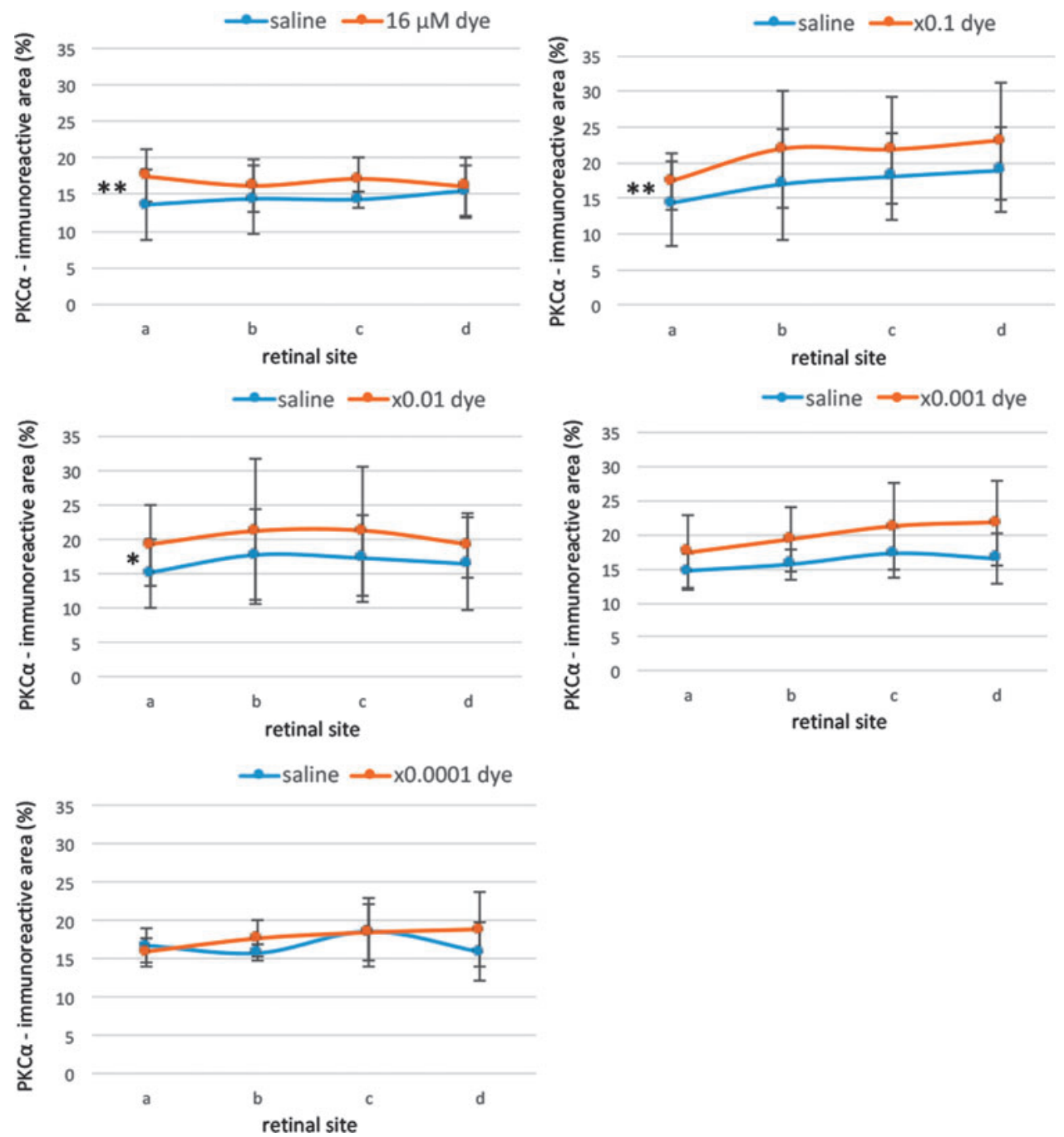

the ganglion cell layer. Because intraocular administration of the photoelectric dye showed inhibitory effect on photoreceptor cell apoptosis, we wondered whether intravitreous dye injection would also prevent rod bipolar cell dysfunction in RCS rats. Thus we performed immunohistochemical staining of $\mathrm{PKC} \alpha$, a rod bipolar cell marker. Dendrites of rod bipolar cells are the major phosphorylation sites of $\mathrm{PKC} \alpha$, and $\mathrm{PKC} \alpha$ upregulation would indicate the improvement of bipolar cell function. ${ }^{31,32}$ Our data revealed that the atrophy of dendrites was correlated with decreased $\mathrm{PKC} \alpha$ immunoreactivity in both dye-injected eyes and saline-injected control eyes. Furthermore, we observed significant increase of $\mathrm{PKC} \alpha$ immunoreactivity in the eyes injected with higher concentrations of the dye. These data suggest that the photoelectric dye may help prevent rod bipolar cell dysfunction in RCS rats.

Previous studies by different groups showed that subretinal implantation of materials could slow down retinal degeneration. ${ }^{5,33,34}$ We initially considered that photoelectric dye-coupled polyethylene films in subretinal space of RCS rats' eyes would exert a similar protective effect on the retina, as described for other materials. This study revealed that the photoelectric dye could slow down the process of retinal degeneration in RCS rats. The therapeutic effect of photoelectric dye on retinal protection, either dependent or independent of light, remains to be determined. Behavioral tests might help evaluate the efficacy of the dye in RCS rats, as performed previously in RCS rats with the dye-coupled film implantation. ${ }^{15,16}$

A major limitation in this study is that we examined the apoptosis by TUNEL assay and the thinning of the outer nuclear layer in the ongoing process of retinal dystrophy at a rather earlier stage in RCS rats. Further studies need to focus on the late stage of retinal dystrophy to test long-term efficacy of the photoelectric dye on overall degeneration of the retinal layers. In addition, it remains unclear how the photoelectric dye prevents photoreceptor degeneration. Further studies are required to elucidate the underlying molecular mechanism of the apoptosis reduction. Semiquantitative measurements of GFAP and PKC $\alpha$ immunoreactivity, as a limitation in the method, would explain inconsistent results in statistical analyses of measurements.

In conclusion, our results show that the injection of the photoelectric dye could effectively reduce the apoptosis of photoreceptor cells and downregulate GFAP expression. In this study, we showed the threshold of the concentration for preventing the photoreceptor degeneration. The best concentration would be obtained from a cell culture experiment 
at the next step. We confirmed biological safety of the photoelectric dye in all tests, including cell safety test, Ames test, chromosomal aberration test, eye irritation test, skin sensitivity test, and acute (a single injection) and subacute (28-day repeated injection) systemic toxicity test (data not shown). The photoelectric dye might be a candidate agent for slowing photoreceptor degeneration. In addition, retinal prosthesis, OUReP, would be positioned as a medical device with pharmacological effect to prevent neuronal apoptosis in the retina.

\section{Acknowledgments}

This study is partly supported by a grant for the Health and Labor Sciences Research from the Ministry of Health, Labor, and Welfare (MHLW) of the Government of Japan, and also by a grant for the Translational Research Network Program from the Japan Agency for Medical Research and Development (AMED).

\section{Author Disclosure Statement}

No competing financial interests exist.

\section{References}

1. Loewenstein, J.I., Montezuma, S.R., and Rizzo, J.F. III. Outer retinal degeneration: an electronic retinal prosthesis as a treatment strategy. Arch. Ophthalmol. 122:587-596, 2004.

2. Tamaki, M., and Matsuo, T. Optical coherence tomographic parameters as objective signs for visual acuity in patients with retinitis pigmentosa, future candidates for retinal prostheses. J. Artif. Organs. 14:140-150, 2011.

3. Zrenner, E. Will retinal implants restore vision? Science. 295:1022-1025, 2002.

4. Humayun, M.S., Dorn, J.D., da Cruz, L., et al. Interim results from the international trial of Second Sight's visual prosthesis. Ophthalmology. 119:779-788, 2012.

5. Chow, A.Y., Chow, V.Y., Packo, K.H., et al. The artificial silicon retina microchip for the treatment of vision loss from retinitis pigmentosa. Arch. Ophthalmol. 122:460-469, 2004.

6. Da Cruz, L., Dorn, J.D., Humayun, M.S., et al. Five-year safety and performance results from the Argus II retinal prosthesis system clinical trial. Ophthalmology. 123:22482254, 2016.

7. Lorach, H., Goetz, G., Smith, R., et al. Photovoltaic restoration of sight with high visual acuity. Nat. Med. 21:476482, 2015.

8. Kelly, S.K., Shire, D.B., Chen, J., et al. A hermetic wireless subretinal neurostimulator for vision prostheses. IEEE Trans. Biomed. Eng. 58:3197-3205, 2011.

9. Matsuo, T., Uchida, T., and Takarabe, K. Safety, efficacy, and quality control of a photoelectric dye-based retinal prosthesis (Okayama University-type retinal prosthesis) as a medical device. J. Artif. Organs. 12:213225, 2009.

10. Matsuo, T. A simple method for screening photoelectric dyes towards their use for retinal prostheses. Acta. Med. Okayama. 57:257-260, 2003.

11. Matsuo, T., Dan-oh Y., and Suga, S. (Inventors). Agent for inducing receptor potential. Assignee: Okayama University. United States Patent. Patent No.: US 7,101,533 B2. Date of Patent: Sep. 5, 2006.
12. Uji, A., Matsuo, T., Ishimaru, S., et al. Photoelectric dyecoupled polyethylene film as a prototype of retinal prostheses. Aritif Organs. 29:53-57, 2005.

13. Uji, A., Matsuo, T., Uchida, T., et al. Intracellular calcium response and adhesiveness of chick embryonic retinal neurons to photoelectric dye-coupled polyethylene films as prototypes of retinal prostheses. Artif. Organs. 30:695-703, 2006.

14. Tamaki, T., Matsuo, T., Hosoya, O., et al. Glial reaction to photoelectric dye-based retinal prostheses implanted in the subretinal space of rats. J. Artif. Organs. 11:38-44, 2008.

15. Alamusi, Matsuo, T., Hosoya, O., et al. Behavior tests and immunohistochemical retinal response analyses in RCS rats with subretinal implantation of Okayama-Universitytype retinal prosthesis. J. Artif. Organs. 16:343-351, 2013.

16. Alamusi, Matsuo, T., Hosoya, O., et al. Vision maintenance and retinal apoptosis reduction in RCS rats with Okayama University-type retinal prosthesis $\left(\mathrm{OUReP}^{\mathrm{TM}}\right)$ implantation. J. Artif. Organs. 18:264-271, 2015.

17. Dowling, J.E., and Sidman, R.L. Inherited retinal dystrophy in the rat. J. Cell. Biol. 14:73-109, 1962.

18. Tso, M.O.M., Zhang, C., Abler, A.S., et al. Apoptosis leads to photoreceptor degeneration in inherited retinal dystrophy of RCS rats. Invest. Ophthalmol. Vis. Sci. 35:2693-2699, 1994.

19. Shen, W.Y., Lai, C.M., Lai, Y.K., et al. Practical considerations of recombinant adeno-associated virus-mediated gene transfer for treatment of retinal degenerations. $J$. Gene. Med. 5:576-587, 2003.

20. Roddy, G.W., and Rosa, R.H., Jr., Oh, J.Y., et al. Stanniocalcin-1 rescued photoreceptor degeneration in two rat models of inherited retinal degeneration. Mol. Ther. 20:788-797, 2012.

21. Faktorovich, E.G., Steinberg, R.H., Yasumura, D., et al. Photoreceptor degeneration in inherited retinal dystrophy delayed by basic fibroblast growth factor. Nature. 347: 83-86, 1990.

22. Hafezi, F., Grimm, C., Simmen, B.C., et al. Molecular ophthalmology: an update on animal models for retinal degenerations and dystrophies. Br. J. Ophthalmol. 84: 922927, 2000.

23. Kolomeyer, A.M., and Zarbin, M.A. Trophic factors in the pathogenesis and therapy for retinal degenerative diseases. Surv. Ophthalmol. 59:134-165, 2014.

24. Sieving, P.A., Caruso, R.C., Tao, W., et al. Ciliary neurotrophic factor (CNTF) for human retinal degeneration: phase I trial of CNTF delivered by encapsulated cell intraocular implants. Proc. Natl. Acad. Sci. U S A. 103:38963901, 2006.

25. Okamoto, K., Matsuo, T., Tamaki, T., et al. Short-term biological safety of a photoelectric dye used as a component of retinal prostheses. J. Artif. Organs. 11:45-51, 2008.

26. Shah, N.V., Pham, D.G., Murray, T.G., et al. Intravitreal and subconjunctival melphalan for retinoblastoma in transgenic mice. J. Ophthalmol. 2014:829879, 2014.

27. Eisenfeld, A.J., Bunt-Milam, A.H., and Sarthy, P.V. Müller cell expression of glial fibrillary acidic protein after genetic and experimental photoreceptor degeneration in the rat retina. Invest. Ophthalmol. Vis. Sci. 25:1321-1328, 1984.

28. Eng, L.F., Ghirnikar, R.S., and Lee, Y.L. Glial fibrillary acidic protein: GFAP-thirty-one years (1969-2000). Neurochem. Res. 25:1439-1451, 2000. 
29. Casola, C., Schiwek, J.E., Reinehr, S., et al. S100 alone has the same destructive effect on retinal ganglion cells as in combination with HSP 27 in an autoimmune glaucoma model. J. Mol. Neurosci. 56:228-236, 2015.

30. Reichelt, W., Dettmer, D., Bruckner, G., et al. Potassium as a signal for both proliferation and differentiation of rabbit retinal (Müller) glia growing in cell culture. Cell Signal. 1:187-194, 1989.

31. Montalbán-Soler, L., Alarcón-Martínez, L., Jiménez-López M., et al. Retinal compensatory changes after light damage in albino mice. Mol. Vis. 18:675-693, 2012.

32. Strettoi, E., Pignatelli, V., Rossi, C., et al. Remodeling of second-order neurons in the retina of $\mathrm{rd} / \mathrm{rd}$ mutant mice. Vision Res. 43:867-877, 2003.

33. Antognazza, M.R., Di Paolo, M., Ghezzi, D., et al. Characterization of a polymer-based, fully organic prosthesis for implantation into the subretinal space of the rat. $A d v$. Healthcare Mater. 10:e201600318, 2016.
34. Fiorani, L., Passacantando, M., Santucci, S., et al. Cerium oxide nanoparticles reduce microglial activation and neurodegenerative events in light damaged retina. PLoS One. 10:e0140387, 2015.

Received: July 11, 2016 Accepted: December 8, 2016

Address correspondence to:

Dr. Toshihiko Matsuo Department of Ophthalmology Okayama University Medical School and Graduate School of Medicine, Dentistry, and Pharmaceutical Sciences 2-5-1 Shikata-cho Okayama City 700-8558

Japan

E-mail: matsuot@cc.okayama-u.ac.jp 